Bull. Korean Math. Soc. 52 (2015), No. 1, pp. 149-157

http://dx.doi.org/10.4134/BKMS.2015.52.1.149

\title{
SHADOWABLE CHAIN COMPONENTS AND HYPERBOLICITY
}

\author{
Manseob Lee, Seunghee Lee, and Junmi Park
}

\begin{abstract}
We show that $C^{1}$-generically, the shadowable chain component of a $C^{1}$-vector field containing a hyperbolic periodic orbit is hyperbolic if it is locally maximal.
\end{abstract}

\section{Introduction}

Chain components and homoclinic classes are central objects in the theory of differentiable dynamical systems since they are natural candidates to replace the Smale's hyperbolic basic sets in non-hyperbolic theory of dynamical systems. Many recent papers have explored their hyperbolic-like properties such as dominated splitting, partial hyperbolicity, etc. For instance, Sakai [9] proved that if the chain component $C_{f}(p)$ of a diffeomorphism $f$ containing a hyperbolic periodic point $p$ is $C^{1}$-robustly shadowable and the $C_{f}(p)$-germ of $f$ is expansive, then $C_{f}(p)$ is hyperbolic. Moreover Wen et al. [11] claimed that the assumption of the $C_{f}(p)$-germ expansivity of $f$ can be dropped in the above result to show the hyperbolicity of the $C^{1}$-robustly shadowable chain component $C_{f}(p)$. However, it is still open whether the above results can be extended to the case of vector fields. More precisely, the first open problem can be formally stated as follows.

Open Problem 1. If the chain component $C_{X}(\gamma)$ of a $C^{1}$-vector field $X$ containing a hyperbolic periodic orbit $\gamma$ is $C^{1}$-robustly shadowable, then is it hyperbolic?

Here, we say that $C_{X}(\gamma)$ is $C^{1}$-robustly shadowable if there exists a neighbor$\operatorname{hood} \mathcal{U}(X)$ of $X$ such that for any $Y \in \mathcal{U}(X), C_{Y}\left(\gamma_{Y}\right)$ is shadowable, where $\gamma_{Y}$ is the continuation of $\gamma$. In relation to the above open problem, very recently, Lee et al. [6] proved that if the chain component $C_{X}(\gamma)$ does not contain a singularity, then the answer of Open Problem 1 is "yes".

In generic view point, as far as we know, there are no interesting results on the hyperbolicity of shadowable chain components. Here we suggest two open

Received November 25, 2013; Revised September 29, 2014.

2010 Mathematics Subject Classification. Primary 37C50; Secondary 34D10.

Key words and phrases. hyperbolic, shadowable, chain component. 
problems in relation to the hyperbolicity of chain components in generic sense. A subset $\mathcal{R} \subset \mathfrak{X}^{1}(M)$ is called residual if it contains a countable intersection of open and dense subsets of $\mathfrak{X}^{1}(M)$. We will say that a property holds generically if there exists a residual subset $\mathcal{R}$ such that any $X \in \mathcal{R}$ has that property.

Open Problem 2. If the chain component $C_{f}(p)$ of a diffeomorphism $f$ containing a hyperbolic periodic point $p$ is $C^{1}$-generically shadowable, then is it hyperbolic?

Open Problem 3. If the chain component $C_{X}(\gamma)$ of a $C^{1}$-vector field $X$ containing a hyperbolic periodic orbit $\gamma$ is $C^{1}$-generically shadowable, then is it hyperbolic?

In this paper, we claim that the answer of Open Problem 3 is "yes" if $C_{X}(\gamma)$ is locally maximal. More precisely, we prove that $C^{1}$-generically, any shadowable chain component of a $C^{1}$-vector field containing a hyperbolic periodic orbit is hyperbolic if it is locally maximal. Note that the chain components may contain singularities.

Let us pass to the main definitions and results. Let $M$ be a compact $C^{\infty}$ Riemannian manifold without boundary. Denote by $\mathfrak{X}^{1}(M)$ the set of $C^{1}$ vector fields on $M$ endowed with the $C^{1}$-topology. Note that every $X \in \mathfrak{X}^{1}(M)$ generates a $C^{1}$-flow $X_{t}: M \times \mathbb{R} \rightarrow M$. We say that a point $p$ in $M$ is periodic if there is $T>0$ such that $X_{T}(p)=p$, and the set of periodic points of $X \in \mathfrak{X}^{1}(M)$ will be denoted by $P(X)$. A point $p \in M$ is said to be a singulairity of $X$ if $X(p)=0$, and the set of singularities of $X$ will be denoted by $\operatorname{Sing}(X)$. Denote by $\operatorname{Crit}(X)=P(X) \cup \operatorname{Sing}(X)$, and the elements of $\operatorname{Crit}(X)$ are called critical elements of $X$.

Let $d$ be the distance induced from the Riemannian structure on $M$. A sequence $\left\{\left(x_{i}, t_{i}\right): x_{i} \in M, t_{i} \geq 1, i \in \mathbb{Z}\right\}$ in $M \times \mathbb{R}$ is called a $\delta$-pseudo orbit (or $\delta$-chain) of $X$ if

$$
d\left(X_{t_{i}}\left(x_{i}\right), x_{i+1}\right)<\delta
$$

for any $i \in \mathbb{Z}$. We say that a compact $X_{t}$-invariant set $\Lambda \subset M$ is shadowable for $X$ if for any $\epsilon>0$, there is $\delta>0$ satisfying the following property: given any $\delta$-pseudo orbit $\left\{\left(x_{i}, t_{i}\right): t_{i} \geq 1, i \in \mathbb{Z}\right\}$ in $\Lambda$, there are a point $y \in M$ and $h \in \operatorname{Rep}(\mathbb{R})$ such that

$$
d\left(X_{h(t)}(y), X_{t-T_{i}}\left(x_{i}\right)\right)<\epsilon, \quad T_{i} \leq t<T_{i+1},
$$

where $T_{i}=t_{0}+t_{1}+\cdots+t_{i}$ for $i>0, T_{i}=0$ for $i=0$, and $T_{i}=-\left(t_{-1}+\right.$ $t_{-2}+\cdots+t_{i}$ ) for $i<0 ; \operatorname{Rep}(\mathbb{R})$ denotes the set of increasing homeomorphisms (reparametrization) $h: \mathbb{R} \rightarrow \mathbb{R}$ with $h(0)=0$.

A point $x \in M$ is said to be chain recurrent if for any $\delta>0$, there exists a $\delta$ pseudo orbit $\left\{\left(x_{i}, t_{i}\right): t_{i} \geq 1\right\}_{i=1}^{n}$ such that $x_{0}=x$ and $d\left(X_{t_{n-1}}\left(x_{n-1}\right), x_{0}\right)<\delta$.

The set of chain recurrent points of $X$ is called the chain recurrent set of $X$, and will be denoted by $C R(X)$. For any $x, y \in M$, we say that $x \sim y$, if for any $\delta>0$ there are a $\delta$-pseudo orbit $\left\{\left(x_{i}, t_{i}\right): t_{i} \geq 1\right\}_{i=1}^{n}$ such that $x_{0}=x$ 
and $d\left(X_{t_{n-1}}\left(x_{n-1}\right), y\right)<\delta$, and a $\delta$-pseudo orbit $\left\{\left(x_{i}^{\prime}, t_{i}^{\prime}\right): t_{i}^{\prime} \geq 1\right\}_{i=1}^{m}$ such that $x_{0}^{\prime}=y$ and $d\left(X_{t_{m-1}^{\prime}}\left(x_{m-1}^{\prime}\right), x\right)<\delta$.

It is easy to see that $\sim$ gives an equivalence relation on the set $C R(X)$. Every equivalence class of $\sim$ is called a chain component of $X$.

A compact invariant set $\Lambda$ of $X$ is called hyperbolic if there are constants $C>0$ and $\lambda>0$ such that the tangent flow $D X_{t}: T_{\Lambda} M \rightarrow T_{\Lambda} M$ leaves a continuous invariant splitting $T_{\Lambda} M=E^{s} \oplus\langle X\rangle \oplus E^{u}$ satisfying

$$
\left\|\left.D X_{t}\right|_{E^{s}(x)}\right\| \leq C e^{-\lambda t} \text { and }\left\|\left.D X_{-t}\right|_{E^{u}(x)}\right\| \leq C e^{-\lambda t}
$$

for any $x \in \Lambda$ and $t>0$, where $\langle X\rangle$ denotes the subspace generated by the vector field $X$. For any hyperbolic periodic orbit $\gamma$ of $X$, we $\operatorname{define} \operatorname{dim}\left(E_{\gamma}^{s}\right)=$ $\operatorname{index}(\gamma)$. Moreover, the sets

$$
\begin{gathered}
W^{s}(\gamma)=\left\{x \in M: X_{t}(x) \rightarrow \gamma \text { as } t \rightarrow \infty\right\} \text { and } \\
W^{u}(\gamma)=\left\{x \in M: X_{t}(x) \rightarrow \gamma \text { as } t \rightarrow-\infty\right\}
\end{gathered}
$$

are said to be the stable manifold and unstable manifold, respectively.

Let $\gamma$ be a hyperbolic periodic orbit of $X$. Denote by $C_{X}(\gamma)$ the chain component of $X$ containing $\gamma$. The homoclinic class of $X$ associated to $\gamma$, denoted by $H_{X}(\gamma)$, is defined as the closure of the transversal intersection of the stable and unstable manifolds of $\gamma$, that is,

$$
H_{X}(\gamma)=\overline{W^{u}(\gamma) \pitchfork W^{s}(\gamma)}
$$

By definition, we can easily see that both $C_{X}(\gamma)$ and $H_{X}(\gamma)$ are closed and $X_{t}$-invariant. Moreover we see that $H_{X}(\gamma) \subset C_{X}(\gamma)$. Bonatti et al. [2] proved the following remarkable results: $C^{1}$-generically, $H_{X}(\gamma)=C_{X}(\gamma)$.

We recall that a compact invariant set $\Lambda \subset M$ is locally maximal if there exists a neighborhood $U$ of $\Lambda$ such that $\bigcap_{t \in \mathbb{R}} X_{t}(U)=\Lambda$. In this paper, we use the terminology "for $C^{1}$-generic $X$ " to express "there is a residual subset $\mathcal{R} \subset \mathfrak{X}^{1}(M)$ such that for any $X \in \mathcal{R} \ldots$..

In this paper, we prove the following main theorem.

Main Theorem. For $C^{1}$-generic $X \in \mathfrak{X}^{1}(M)$, the chain component $C_{X}(\gamma)$ is hyperbolic if and only if it is shadowable and locally maximal.

\section{Proof of Main Theorem}

Let $\Lambda$ be a closed, $X_{t}$-invariant subset of $M$. If $\Lambda$ is locally maximal, then there exists $\epsilon>0$ such that $\bigcap_{t \in \mathbb{R}} X_{t}\left(B_{\epsilon}(\Lambda)\right)=\Lambda$, where $B_{\epsilon}(\Lambda)$ is the $\epsilon$-neighborhood of $\Lambda$. Suppose $\Lambda$ is shadowable. Then there exists $\delta>0$ such that if $\xi=\left\{\left(x_{i}, t_{i}\right): t_{i} \geq 1, i \in \mathbb{Z}\right\}$ is a $\delta$-pseudo orbit in $\Lambda$, then there are $y \in M$ and $h \in \operatorname{Rep}(\mathbb{R})$ satisfying

$$
d\left(X_{h(t)}(y), X_{t-T_{i}}\left(x_{i}\right)\right)<\epsilon,
$$


where $T_{i} \leq t<T_{i+1}$ for all $i \in \mathbb{Z}$. Then we have

$$
\mathcal{O}(y) \subset \bigcap_{t \in \mathbb{R}} X_{t}\left(B_{\epsilon}(\Lambda)\right)=\Lambda \text {. }
$$

This means that the shadowing point $y$ of the $\delta$-pseudo orbit $\xi=\left\{\left(x_{i}, t_{i}\right): t_{i} \geq\right.$ $1, i \in \mathbb{Z}\}$ in $\Lambda$ can be taken from $\Lambda$. Let $\gamma$ be a hyperbolic periodic orbit of $X$, and let $p \in \gamma$. Then there is $\epsilon=\epsilon(p)>0$ such that

$$
\begin{gathered}
W_{\epsilon}^{s}(p)=\left\{y \in M: d\left(X_{t}(y), X_{t}(p)\right)<\epsilon, t \geq 0\right\}, \text { and } \\
W_{\epsilon}^{u}(p)=\left\{y \in M: d\left(X_{t}(y), X_{t}(p)\right)<\epsilon, t \leq 0\right\}
\end{gathered}
$$

are $C^{1}$-embedded submanifolds of $M$. Clearly we have $W_{\epsilon}^{s}(p) \subset W^{s}(\gamma)$ and $W_{\epsilon}^{u}(p) \subset W^{u}(\gamma)$.

Lemma 2.1. Let $\Lambda$ be a transitive set of $M$. Suppose $\Lambda$ is shadowable. Then for any hyperbolic critical elements $\eta, \tau$ in $\Lambda$, we have

$$
W^{s}(\eta) \cap W^{u}(\tau) \neq \emptyset \text { and } W^{u}(\eta) \cap W^{s}(\tau) \neq \emptyset .
$$

Proof. Let $\eta, \tau$ be hyperbolic critical elements in $\Lambda$, and take $p \in \eta$ and $q \in \tau$. (If $\eta$ or $\tau$ is singularity, we can put $p=\eta$ or $q=\tau$, respectively.) Choose $\epsilon(p)>0$ and $\epsilon(q)>0$ such that $W_{\epsilon(p)}^{s}(p), W_{\epsilon(p)}^{u}(p), W_{\epsilon(q)}^{s}(q)$, and $W_{\epsilon(q)}^{u}(q)$ are well-defined. Let $\epsilon=\min \{\epsilon(p), \epsilon(q)\}$, and take a constant $0<\delta<\epsilon$ corresponding to $\epsilon$ by the shadowing property of $\Lambda$. Since $\Lambda$ is transitive, we can take $x \in \Lambda$ and constants $l, m>0$ such that

$$
d\left(X_{l}(x), p\right)<\delta \text { and } d\left(X_{l+m}(x), q\right)<\delta .
$$

Construct a $\delta$-pseudo orbit $\xi=\left\{\left(x_{i}, t_{i}\right): t_{i}=1\right\}_{i \in \mathbb{Z}}$ in $\Lambda$ containing $p$ and $q$ as follows:

$$
\left\{\begin{aligned}
x_{-i} & =X_{-i}(p) & & \text { for } \quad i \geq 1 \\
x_{i} & =X_{l+i}(x) & & \text { for } \quad 0 \leq i \leq m-1 \\
x_{m+i} & =X_{i}(q) & & \text { for } \quad i \geq 0 .
\end{aligned}\right.
$$

Then there is $z \in M$ and $h \in \operatorname{Rep}(\mathbb{R})$ such that

$$
d\left(X_{h(t)}(z), X_{t-T_{i}}\left(x_{i}\right)\right)<\epsilon
$$

for all $i \in \mathbb{Z}, T_{i} \leq t<T_{i+1}$. Since $t(1-\epsilon)<h(t)<t(1+\epsilon)$ and $T_{i} \leq t<T_{i+1}$, we know that $h(t) \rightarrow \pm \infty$ as $t \rightarrow \pm \infty$ which implies that

$$
d\left(X_{h(t)}(z), X_{t-T_{i}}\left(x_{i}\right)\right)=d\left(X_{h(t)}(z), X_{t-T_{i}}(q)\right)<\epsilon
$$

for $T_{i} \leq i<T_{i+1}$ for all $i \geq m$, and $d\left(X_{t}(z), X_{t}(p)\right)<\epsilon$ for $t \leq 0$. This means that $X_{t}(z) \in W_{\epsilon}^{s}(q)$ for $t>T_{m}$, and so, $z \in W^{s}(\tau)$, and $z \in \bar{W}_{\epsilon}^{u}(p) \subset W^{u}(\eta)$. Thus we can conclude that $W^{u}(\eta) \cap W^{s}(\tau) \neq \emptyset$. Similarly, we can show that $W^{s}(\eta) \cap W^{u}(\tau) \neq \emptyset$. 
Note that, we can apply Lemma 2.1 to the homoclinic class $H_{X}(\gamma)$ since the homoclinic class $H_{X}(\gamma)$ is transitive. A vector field $X \in \mathfrak{X}^{1}(M)$ is said to be Kupka-Smale if any element of $\operatorname{Crit}(X)$ is hyperbolic and its invariant manifolds intersect transversely. It is well known that the set of Kupka-Smale vector fields, denoted by $\mathcal{K S}(M)$, is residual in $\mathfrak{X}^{1}(M)$.

Lemma 2.2. Let $X \in \mathcal{K S}(M)$, and let $\gamma$ be a hyperbolic periodic orbit of $X$. If $\gamma_{1}, \gamma_{2}$ are hyperbolic critical elements in $H_{X}(\gamma)$ with $\operatorname{dim} W^{s}\left(\gamma_{1}\right)+$ $\operatorname{dim} W^{u}\left(\gamma_{2}\right) \leq \operatorname{dim} M$, then $W^{s}\left(\gamma_{1}\right) \cap W^{u}\left(\gamma_{2}\right)=\emptyset$.

Proof. See [1, Lemma 3.4].

Lemma 2.3. There is a residual subset $\mathcal{R}_{1}$ of $\mathfrak{X}^{1}(M)$ such that for any hyperbolic periodic orbit $\gamma$ of $X \in \mathcal{R}_{1}$, if the chain component $C_{X}(\gamma)$ is shadowable, locally maximal and $W^{s}(\eta) \cap W^{u}(\tau) \neq \emptyset$ for any critical elements $\eta, \tau$ in $C_{X}(\gamma)$, then $X$ has no singularities in $C_{X}(\gamma)$.

Proof. Take a residual subset $\tilde{\mathcal{R}}$ of $\mathfrak{X}^{1}(M)$ such that for any $X \in \tilde{\mathcal{R}}$ and a hyperbolic periodic orbit $\gamma$ of $X$, we have $C_{X}(\gamma)=H_{X}(\gamma)$. Let $\mathcal{R}_{1}=\tilde{\mathcal{R}} \cap$ $\mathcal{K} \mathcal{S}(M)$, and let $\gamma$ be a hyperbolic periodic orbit of $X \in \mathcal{R}_{1}$ with index $j$. Suppose that $X$ has a hyperbolic singularity $\sigma$ in $H_{X}(\gamma)$ with index $i$. By [8, Lemma 7], if $j>i$, then

$$
\operatorname{dim} W^{u}(\sigma)+\operatorname{dim} W^{s}(\gamma)<\operatorname{dim} M
$$

This is a contradiction since $X$ is Kupka-Smale. Similarly, we can get a same contradiction for the case of $j<i$. This completes the proof.

From Lemma 2.3, for $C^{1}$-generic vector field $X \in \mathfrak{X}^{1}(M)$, if $C_{X}(\gamma)$ is shadowable and locally maximal, we can see that there is no singularity for $X$.

Proposition 2.4. There is a residual subset $\mathcal{R}_{2}$ of $\mathfrak{X}^{1}(M)$ such that for any hyperbolic periodic orbit $\gamma$ of $X \in \mathcal{R}_{2}$, if the chain component $C_{X}(\gamma)$ is shadowable and locally maximal, then $\operatorname{ind} \operatorname{dex}(\eta)=\operatorname{index}(\tau)$ for any hyperbolic periodic orbits $\eta, \tau$ in $C_{X}(\gamma)$.

Proof. Take a residual subset $\mathcal{R}_{2}=\mathcal{R}_{1}$ of $\mathfrak{X}^{1}(M)$, and let for any hyperbolic periodic orbit $\gamma$ of $X \in \mathcal{R}_{2}, C_{X}(\gamma)$ be shadowable and two hyperbolic periodic orbits of $X$ in $C_{X}(\gamma)$ be saddles. Suppose that $\operatorname{index}(\eta) \neq \operatorname{index}(\tau)$. Then we have

$$
\operatorname{dim} \mathrm{W}^{\mathrm{s}}(\eta)+\operatorname{dim} \mathrm{W}^{\mathrm{u}}(\tau) \leq \operatorname{dim} \mathrm{M} .
$$

Since $X \in \mathcal{K} \mathcal{S}(M)$, by Lemma $2.2, W^{s}(\eta) \cap W^{u}(\tau)=\emptyset$. This is a contradiction to Lemma 2.1.

Let $X \in \mathfrak{X}^{1}(M), p \in M$ a point in a periodic orbit of $X_{t}$ with period $T>0$ and $T_{p} M(s)=\left\{v \in T_{p} M:\|v\| \leq s\right\}$. Define $\langle X(p)\rangle$ as the subspace generated by $X(p)$, and set

$$
N_{p}=\langle X(p)\rangle^{\perp} \text { and } N_{p, s}=N_{p} \cap T_{p} M(s) \text { for } 0<s<1
$$


such that the exponential map $\exp _{p}: T_{p} M(s) \rightarrow M$ is well defined for all $p \in M$. Finally define $\mathcal{N}_{p, s}=\exp _{p}\left(N_{p, s}\right)$. Then for a given $p^{\prime}=X_{t_{0}}(p)$ with $t_{0}>0$, there are $r_{0}>0$ and a $C^{1}$ map defined by

$$
\tau: \mathcal{N}_{p, r_{0}} \rightarrow \mathbb{R} \text { such that } X_{\tau(y)}(y) \in \mathcal{N}_{p^{\prime}, s}
$$

for all $y \in \mathcal{N}_{p, r_{0}}$ with $\tau(p)=t_{0}$.

The flow $X_{t}$ uniquely defines the Pincaré map

$$
\begin{array}{cl}
f: \mathcal{N}_{p, r_{0}} & \rightarrow \mathcal{N}_{p^{\prime}, s}, \\
y & \mapsto X_{\tau(y)}(y) .
\end{array}
$$

This map is a $C^{1}$ embedding whose image set is contained in the interior of $\mathcal{N}_{p^{\prime}, s}$ if $r_{0}$ is small. If $X_{t}(p) \neq p$ for $0<t \leq t_{0}$ and $r_{0}$ is sufficiently small, then the map $(t, y) \mapsto X_{t}(y)$ is a $C^{1}$ embedding from the set $\left\{(t, y) \in \mathbb{R} \times \mathcal{N}_{p, r}: 0 \leq\right.$ $t \leq \tau(y)\}$ into $M$ for $0<r \leq r_{0}$. The image will be denoted by

$$
F_{p}\left(X_{t}, r, t_{0}\right)=\left\{X_{t}(y): y \in \mathcal{N}_{p, r} \text { and } 0 \leq t \leq \tau(y)\right\} .
$$

For $\epsilon>0$, let $U_{\epsilon}\left(\mathcal{N}_{p, r}\right)$ be the set of diffeomorphisms $\varphi: \mathcal{N}_{p, r} \rightarrow \mathcal{N}_{p, r}$ such that $\operatorname{supp}(\varphi) \subset \mathcal{N}_{p, r / 2}$ and $d_{1}(\varphi, i d)<\epsilon$. Here $d_{1}$ is the usual $C^{1}$ metric, $i d: \mathcal{N}_{p, r} \rightarrow \mathcal{N}_{p, r}$ is the identity map, and $\operatorname{supp}(\varphi)$ is the closure of the set where it differs from $i d$. Note that $p$ is a hyperbolic fixed point of $f$ if and only if its orbit $O(p)$ is hyperbolic.

Lemma 2.5. Let $X \in \mathfrak{X}^{1}(M), p$ a periodic point of $X$ with period $T>0$, let $f: \mathcal{N}_{p, r_{0}} \rightarrow \mathcal{N}_{p, s}$ be as above, and let $\mathcal{U} \subset \mathfrak{X}^{1}(M)$ be a $C^{1}$-neighborhood of $X$ and $0<r \leq r_{0}$ be given. Then there are $\delta_{0}>0$ and $0<\epsilon_{0}<r / 2$ such that for a linear isomorphism $L_{\delta}: N_{p} \rightarrow N_{p}$ with $\left\|L_{\delta}-D_{p} f\right\|<\delta<\delta_{0}$, there is $Y^{\delta} \in \mathcal{U}$ satisfying:

(1) $Y^{\delta}(x)=X(x)$, if $x \notin F_{t}\left(X_{t}, r, T\right)$,

(2) $p$ belongs to a periodic orbit for $Y_{t}^{\delta}$,

(3)

$$
g_{Y^{\delta}}(x)= \begin{cases}\exp _{p} \circ H_{\delta} \circ \exp _{p}^{-1}(x) & \text { if } x \in B_{\epsilon_{0} / 4}(p) \cap \mathcal{N}_{p, r} \\ f(x) & \text { if } x \notin B_{\epsilon_{0}}(p) \cap \mathcal{N}_{p, r}\end{cases}
$$

where $g_{Y^{\delta}}(x): \mathcal{N}_{p, r} \rightarrow \mathcal{N}_{p, s}$ is the Poincaré map of $Y_{t}^{\delta}$. Furthermore, let $Y^{0}$ be the vector field for $H_{0}=D_{p} f$. Then we have

(4) $d_{1}\left(Y^{\delta}, Y^{0}\right) \rightarrow 0$ as $\delta \rightarrow 0$

Proof. See [7, Lemma 1.3, p. 3395].

Lemma 2.6. There is a residual subset $\mathcal{R}_{3}$ of $\mathfrak{X}^{1}(M)$ such that for any $X \in$ $\mathcal{R}_{3}, X$ satisfies the following: let $C_{X}(\gamma)$ be shadowable and locally maximal in $U$ for a hyperbolic periodic orbit $\gamma$ of $X \in \mathcal{R}_{3}$, and let $\mathcal{U}(X)$ be a $C^{1}$-neighborhood of $X$. If a periodic orbit of $X$ in $C_{X}(\gamma)$ is not hyperbolic, then there is $Y \in$ $\mathcal{U}(X)$ such that $Y$ has two hyperbolic periodic orbits $\eta_{Y}, \tau_{Y} \in C_{Y}\left(\gamma_{Y}\right) \subset U$ with $\operatorname{index}\left(\eta_{Y}\right) \neq \operatorname{index}\left(\tau_{Y}\right)$. Here, $\gamma_{Y}$ is the continuation of $\gamma$ for $Y$. 
Proof. Since, by [3], for any $X \in \mathcal{R}_{3}, C_{X}(\gamma)$ is robustly isolated. So, there exist a neighborhood $\mathcal{U}(X)$ of $X$ and a neighborhood $U$ of $C_{X}(\gamma)$ such that for any $Y \in \mathcal{U}(X)$,

$$
C_{Y}\left(\gamma_{Y}\right)=\Lambda_{Y}(U)\left(=\bigcap_{t \in \mathbb{R}} Y_{t}(U)\right) .
$$

Therefore we can apply the result of [1, Lemma 4.3].

Note that since $C_{X}(\gamma)$ is locally maximal in an open set $U$ we know that $\eta_{Y}, \tau_{Y} \in C_{Y}\left(\gamma_{Y}\right) \subset U$.

Lemma 2.7. There is a residual subset $\mathcal{R}_{4}$ of $\mathfrak{X}^{1}(M)$ such that for any $X \in$ $\mathcal{R}_{4}$, if for any $C^{1}$-neighborhood $\mathcal{U}(X)$ of $X$, there exists $Y \in \mathcal{U}(X)$ such that $Y$ has two hyperbolic periodic orbits $\eta_{Y}, \tau_{Y}$ with $\operatorname{index}\left(\eta_{Y}\right) \neq \operatorname{index}\left(\tau_{Y}\right)$, then $X$ has two hyperbolic periodic orbits $\eta, \tau$ with $\operatorname{index}(\eta) \neq \operatorname{index}(\tau)$.

Proof. See, [1, Lemma 5.1].

We say that a vector field $X$ is a star vector field if there exists a $C^{1}$ neighborhood $\mathcal{U}(X)$ of $X$ in $\mathfrak{X}^{1}(M)$ such that for any $Y \in \mathcal{U}(X)$, every critical element of $Y$ is hyperbolic, and $M$ is said to satisfy the star condition for $X$ if $X$ is a star vector field. Moreover, we say that a compact invariant set $\Lambda \subset M$ satisfies the star condition for $X \in \mathfrak{X}^{1}(M)$ if there exist a $C^{1}$-neighborhood $\mathcal{U}(X)$ of $X$ in $\mathfrak{X}^{1}(M)$ and a neighborhood $U$ of $\Lambda$ such that for any $Y \in \mathcal{U}(X)$, every critical element of $Y$ in $\Lambda_{Y}(U)=\bigcap_{t \in \mathbb{R}} Y_{t}(U)$ is hyperbolic.

Proposition 2.8. There is a residual subset $\mathcal{R}^{\prime}$ of $\mathfrak{X}^{1}(M)$ such that for any hyperbolic periodic orbit $\gamma$ of $X \in \mathcal{R}^{\prime}$, if $C_{X}(\gamma)$ is shadowable and locally maximal, then $C_{X}(\gamma)$ satisfies the star condition.

To prove Proposition 2.8, we need the notion of a $\delta$-weak eigenvalue (see [10]). Recall that if $p$ is in a periodic orbit of $X$ with period $\pi(p)$, then $D X_{\pi(p)}(p)$ has 1 as eigenvalue with eigenvector $X(p)$, and all the other eigenvalues are called the characteristic multipliers of $p$. We say that a point $p$ in a hyperbolic periodic orbit of $X$ has a $\delta$-weak eigenvalue if there is a characteristic multiplier $\sigma$ of the orbit of $p$ such that

$$
(1-\delta)<|\delta|<(1+\delta)
$$

Lemma 2.9. There is a residual subset $\mathcal{R}_{5}$ of $\mathfrak{X}^{1}(M)$ such that for any hyperbolic periodic orbit $\gamma$ of $X \in \mathcal{R}_{5}$, if the chain component $C_{X}(\gamma)$ is shadowable and locally maximal, then there is $\delta>0$ such that every periodic orbit in $C_{X}(\gamma)$ does not have a $\delta$-weak eigenvalue.

Proof. Take a residual subset $\mathcal{R}_{5}=\mathcal{R}_{2} \cap \mathcal{R}_{3} \cap \mathcal{R}_{4}$, and let $C_{X}(\gamma)$ is shadowable and locally maximal for any hyperbolic periodic orbit $\gamma$ of $X \in \mathcal{R}_{5}$. To derive a contradiction, we may assume that there is a point $p$ which belongs to a periodic orbit $\eta$ in $C_{X}(\gamma)$ such that for any $\delta>0, p$ has a $\delta$-weak eigenvalue. Then by Lemmas 2.5 and 2.6, there is $Y C^{1}$-close to $X$ such that $Y$ has two hyperbolic 
periodic orbits $\eta_{Y}, \tau_{Y}$ in $\Lambda_{Y}(U)=\bigcap_{t \in \mathbb{R}} Y_{t}(U)$ with $\operatorname{index}\left(\eta_{Y}\right) \neq \operatorname{index}\left(\tau_{Y}\right)$. Since $C_{X}(\gamma)$ is locally maximal, by Lemma $2.7, X$ has two hyperbolic periodic orbits $\eta, \tau$ in $C_{X}(\gamma)$ with $\operatorname{index}(\eta) \neq \operatorname{index}(\tau)$. This contradicts to Proposition 2.4 .

Proof of Proposition 2.8. Take a residual subset $\mathcal{R}^{\prime}=\mathcal{R}_{5}$ and suppose that for any hyperbolic periodic orbit $\gamma$ of $X \in \mathcal{R}^{\prime}, C_{X}(\gamma)$ dose not satisfy the star condition. Then we may assume that there is a periodic point $p$ in $C_{X}(\gamma)$ such that for any $\delta>0, p$ has a $\delta$-weak eigenvalue. This is a contradiction to Lemma 2.9 .

Let $P O_{h}(X)$ be the set of hyperbolic periodic orbits of $X$.

Proposition 2.10. Let $\gamma \in P O_{h}(X)$ and let $T \geq 1, \eta>0$ and $\tilde{T}>0$ be given. For any $\gamma^{\prime} \sim \gamma$, if the period $\tau$ of $\gamma^{\prime}$ is bigger than $\tilde{T}$, we assume $H_{X}(\gamma)$ satisfies the following properties (P1) to (P3):

(P1) For any $x \in \gamma^{\prime}$ and $t \geq T$,

$$
\frac{1}{t}\left(\log \left\|\left.\Psi_{t}\right|_{\triangle^{s}(x)}\right\|-\log m\left(\left.\Psi_{t}\right|_{\triangle^{u}(x)}\right)\right)<-2 \eta .
$$

(P2) Let $x \in \gamma^{\prime}$ and $0=T_{0}<T_{1}<\cdots<T_{\iota}=\tau$ be a partition with $T \leq T_{i}-T_{i-1}<2 T$ for any $i=1, \ldots, \iota$. Then we have

$$
\begin{aligned}
& \left.\frac{1}{\tau} \sum_{i=1}^{i=\iota} \log || \Psi_{T_{i}-T_{i-1}}\right|_{\triangle^{s}\left(X_{T_{i-1}} x\right)} \|<-\eta, \\
& \frac{1}{\tau} \sum_{i=1}^{i=\iota} \log m\left(\left.\Psi_{T_{i}-T_{i-1}}\right|_{\triangle^{u}\left(X_{T_{i-1}} x\right)}\right)>\eta .
\end{aligned}
$$

(P3) $X$ has the shadowing property on $H_{X}(\gamma)$.

Then $H_{X}(\gamma)$ is hyperbolic.

Proof. See [5].

Proof of Main Theorem. Take a residual subset $\mathcal{R}=\mathcal{R}^{\prime}$ of $\mathfrak{X}^{1}(M)$ and suppose that for any hyperbolic periodic orbit $\gamma$ of $X \in \mathcal{R}, C_{X}(\gamma)$ is shadowable and locally maximal. Then by Proposition 2.8, $C_{X}(\gamma)$ satisfies the star condition. If we apply the result [4, Lemma VII.1], we can see that $X$ satisfies (P1), (P2) of Proposition 2.10. This completes the proof by Proposition 2.10.

Acknowledgments. ML is supported by Basic Science Research Program through the National Research Foundation of Korea(NRF) funded by the Ministry of Science, ICT \& Future Planning (No. 2014R1A1A1A05002124). SL was supported by BK21 plus math vision 2020 project. 


\section{References}

[1] A. Arbieto, L. Senos, and T. Sodero, The specification property for flows from the robust and generic viewpoint, J. Differential Equations 253 (2012), no. 1, 1893-1909.

[2] C. Bonatti and S. Crovisier, Récurrence et généricité, Invent. Math. 158 (2004), no. 1, 33-104.

[3] C. Bonatti and L. Diaz, Robust heterodimensional cycles and $C^{1}$-generic dynamics, J. Inst. Math. Jussieu 7 (2008), no. 3, 469-525.

[4] S. Hayashi, Connecting invariant manifolds and the solution of the $C^{1}$ stability and S-stability conjectures for flows, Ann. of Math. 145 (1997), no. 1, 81-137.

[5] K. Lee, M. Lee, and S. Lee, Hyperbolicity of expansive homoclinic classes, preprint.

[6] K. Lee, L. Tien, and X. Wen, Robustly shadowable chain component of $C^{1}$-vector fields, J. Korean Math. Soc. 51 (2014), no. 1, 17-53.

[7] K. Moriyasu, K. Sakai, and N. Sumi, Vector fields with topological stability, Trans. Amer. Math. Soc. 353 (2001), no. 8, 3391-3408.

[8] R. Ribeiro, Hyperbolicity and types of shadowing for $C^{1}$ generic vector fields, arXiv: $1305.2977 \mathrm{v} 1$

[9] K. Sakai, $C^{1}$-stably shadowable chain components, Ergodic Theory Dynam. Systems 28 (2008), no. 3, 987-1029.

[10] L. Senos, Generic Bowen-expansive flows, Bull. Braz. Math. Soc. 43 (2012), no. 1, 59-71.

[11] L. Wen, S. Gan, and X. Wen, $C^{1}$-stably shadowable chain components are hyperbolic, J. Differential Equations 236 (2009), no. 1, 340-357.

Manseob Lee

Department of Mathematics

MOKWON UNIVERSITY

DAEJEON 302-729, KOREA

E-mail address: lmsds@mokwon.ac.kr

Seunghee Lee

Department of Mathematics

Chungnam National University

DAEJEON 305-764, KorEA

E-mail address: shlee@cnu.ac.kr

JUNMI PARK

Department of Mathematics

Chungnam National University

DAEJEON 305-764, KOREA

E-mail address: pjmds@cnu.ac.kr 